\title{
EIGENVALUES OF POLYHARMONIC OPERATORS ON VARIABLE DOMAINS
}

\author{
Davide Buoso $^{1}$ and Pier Domenico Lamberti ${ }^{1}$
}

\begin{abstract}
We consider a class of eigenvalue problems for polyharmonic operators, including Dirichlet and buckling-type eigenvalue problems. We prove an analyticity result for the dependence of the symmetric functions of the eigenvalues upon domain perturbations and compute Hadamard-type formulas for the Frechét differentials. We also consider isovolumetric domain perturbations and characterize the corresponding critical domains for the symmetric functions of the eigenvalues. Finally, we prove that balls are critical domains.
\end{abstract}

Mathematics Subject Classification. 35J40, 35B20, 35P15.

Received April 4, 2012. Revised November 13, 2012.

Published online September 6, 2013.

\section{INTRODUCTION}

Let $n, m \in \mathbb{N}_{0}$ with $0 \leq m<n$ and $\Omega$ be a bounded open set in $\mathbb{R}^{N}$ with smooth boundary. We consider the following eigenvalue problem

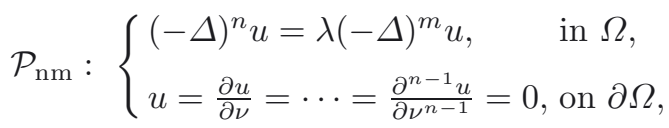

where $\nu$ denotes the unit outer normal to $\partial \Omega$. The case $m=0$ corresponds to the well-known eigenvalue problem for the polyharmonic operator $(-\Delta)^{n}$ subject to Dirichlet boundary conditions, while the case $m>0$ represents a buckling-type problem. These cases include important problems in linear elasticity. For instance, for $N=2$, $\mathcal{P}_{10}$ arises in the study of a vibrating membrane stretched in a fixed frame, $\mathcal{P}_{20}$ corresponds to the case of a vibrating clamped plate and $\mathcal{P}_{21}$ is related to plate buckling. We are mainly interested in the Dirichlet problem $\mathcal{P}_{\text {n0 }}$ and the buckling problem $\mathcal{P}_{21}$. However, we prefer to present a unified approach involving all cases. Problem $\mathcal{P}_{\mathrm{nm}}$ admits a divergent sequence of positive eigenvalues of finite multiplicity represented as follows

$$
0<\lambda_{1}[\Omega] \leq \lambda_{2}[\Omega] \leq \cdots \leq \lambda_{j}[\Omega] \leq \cdots .
$$

As usual, we agree to repeat each eigenvalue as many times as its multiplicity.

\footnotetext{
Keywords and phrases. Polyharmonic operators, eigenvalues, domain perturbation.

1 Dipartimento di Matematica, Università degli Studi di Padova, Via Trieste, 63, 35126 Padova, Italy.

dbuoso@math.unipd.it; lamberti@math.unipd.it
} 
In this paper we are interested in the dependence of $\lambda_{j}[\Omega]$ on $\Omega$. There is a vast literature devoted to domain perturbation problems for elliptic operators. In particular, the cases $n=1,2$ which correspond to the Laplace operator and the biharmonic operator respectively, have been intensively studied by many authors. We refer to Bucur and Buttazzo [4], Daners [10], Hale [13], Henry [15], Henrot [14], Kesavan [18] for updated information on this topic. The case $n>2$ has been much less investigated. However, a renewed general interest in higher order operators has been growing in the last decades as it appears in the extensive monograph by Gazzola, Grunau and Sweers [11] devoted to recent developments in the theory of polyharmonic operators. As for domain perturbation problems, we refer to the papers $[5,6]$ where spectral stability estimates for elliptic operators of arbitrary order are proved. See also the survey paper [7].

Our work is inspired by classical problems in spectral optimization, in particular by the celebrated Rayleigh conjecture (see e.g., [14]). Recall that the Rayleigh-Faber-Krahn inequality states that the first eigenvalue of the Laplace operator with Dirichlet boundary conditions (problem $\mathcal{P}_{10}$ ) is minimized by the ball in the class of all bounded domains with a fixed measure. In symbols,

$$
\lambda_{1}\left[\Omega^{*}\right] \leq \lambda_{1}[\Omega]
$$

where $\Omega^{*}$ is a ball with the same measure of $\Omega$. As for the biharmonic operator with Dirichlet boundary conditions (problem $\mathcal{P}_{20}$ ), inequality (1.2) was proved by Nadirashvili [23] for $N=2$ and by Ashbaugh and Benguria [2] for $N=3$. See also Mohr [22]. Regarding the buckling of a plate (problem $\mathcal{P}_{21}$ ), Szegö $[26,27]$ proved inequality (1.2) under the restrictive assumption that the first eigenfunction has constant sign. Such assumption was not used by Willms and Weinberger who proved inequality (1.2) for $N=2$ by assuming existence and regularity of the minimizing domain. That proof was presented by Willms in [28] and is described in Kawohl [17]. Note that the existence of the minimizer in the class of simply connected open sets in the plane was later proved by Ashbaugh and Bucur [3], whilst the regularity of such minimizer seems to be an open problem.

It should be noted that not much is known for higher eigenvalues $\lambda_{j}[\Omega]$ for $j>2$. As a corollary of a general result by Buttazzo and Dal Maso [8], it is known that each eigenvalue of the Dirichlet Laplacian admits a minimizer in the class of all quasi-open sets with fixed measure, contained in a prescribed bounded region. However, that result says little about the shape of such minimizer. It is proved in Wolf and Keller [29] that the minimizers of higher eigenvalues in general are not balls and not even unions of balls. Moreover, by looking at the interesting numerical results presented in Oudet [25], one may get the idea that balls are not much relevant in the analysis of higher eigenvalues.

Our main aim is to point out that, despite the above mentioned negative results, balls play a relevant role in the study of isovolumetric perturbations of $\Omega$ for all eigenvalues $\lambda_{j}[\Omega]$ of all problems $\mathcal{P}_{\text {nm }}$. To do so we shall consider problems $\mathcal{P}_{\mathrm{nm}}$ on families of open sets $\phi(\Omega)$ described by suitable diffeomorphisms $\phi$ defined on a fixed open set $\Omega$ and we shall study the dependence of $\lambda_{j}[\phi(\Omega)]$ on $\phi$. As is known, this allows to talk about differentiability and apply calculus in order to find critical eigenvalues with respect to perturbations of $\phi$. One of the main difficulties in the analysis of higher eigenvalues is related to the variation of their multiplicity. This leads to bifurcation phenomena which complicate things. For instance, if a fixed open set $\tilde{\phi}(\Omega)$ is subject to a perturbation $\phi$ of $\tilde{\phi}$ then a multiple eigenvalue $\lambda_{j}[\tilde{\phi}(\Omega)]$ of multiplicity $r$ may split into $r$ simple eigenvalues $\lambda_{j}[\phi(\Omega)], \ldots, \lambda_{j+r-1}[\phi(\Omega)]$ in such a way that $\lambda_{j}[\phi(\Omega)], \ldots, \lambda_{j+r-1}[\phi(\Omega)]$ are not differentiable in the variable $\phi$. As for the Laplace operator with Dirichlet or Neumann boundary conditions, it was pointed out in [19] that in the case of multiple eigenvalues it is natural to consider the elementary symmetric functions of the eigenvalues $\lambda_{j}[\phi(\Omega)], \ldots, \lambda_{j+r-1}[\phi(\Omega)]$. In this paper, we generalize the results of $[19,20]$ to all problems $\mathcal{P}_{\mathrm{nm}}$. Namely, we prove that the elementary symmetric functions of the eigenvalues $\lambda_{j}[\phi(\Omega)], \ldots, \lambda_{j+r-1}[\phi(\Omega)]$ of $\mathcal{P}_{\mathrm{nm}}$ depend real analytically on $\phi$ (Thm. 3.1) and we prove that if $\tilde{\phi}(\Omega)$ is a ball then $\tilde{\phi}$ is a critical point for such functions under volume constraint (Thm. 4.2). In fact, all critical points $\tilde{\phi}$ for the symmetric functions of the eigenvalues splitting from an eigenvalue $\lambda$ of multiplicity $r$, can be characterized as those open sets for which the following 
overdetermined system has a nontrivial solution $\left(u_{1}, \ldots, u_{r}\right)$

$$
\begin{cases}(-\Delta)^{n} u_{i}=\lambda(-\Delta)^{m} u_{i}, & \text { in } \tilde{\phi}(\Omega), \quad \forall i=1, \ldots, r, \\ u_{i}=\frac{\partial u_{i}}{\partial \nu}=\cdots=\frac{\partial^{n-1} u_{i}}{\partial \nu^{n-1}}=0, & \text { on } \partial \tilde{\phi}(\Omega), \forall i=1, \ldots, r, \\ \left(\frac{\partial^{n} u_{1}}{\partial \nu^{n}}\right)^{2}+\cdots+\left(\frac{\partial^{n} u_{r}}{\partial \nu^{n}}\right)^{2}=\text { const }, & \text { on } \partial \tilde{\phi}(\Omega) .\end{cases}
$$

Since (1.3) is satisfied if $\tilde{\phi}(\Omega)$ is a ball, it would be interesting to clarify whether the existence of nontrivial solutions to (1.3) on a bounded connected open set $\tilde{\phi}(\Omega)$ implies that $\tilde{\phi}(\Omega)$ is a ball. For $r=1, n=1, m=0$ or $n=2, m=0$ this can be proved by using the celebrated moving plane method under the assumption that the solution does not change sign (see e.g., Henry [16] for the Laplace operator and Dalmasso [9] for the biharmonic operator); for $r=1, n=2, m=1$ the method by Willms and Weinberger leads to the same conclusion (see e.g., $[14,17])$.

\section{Notation AND PRELIMINARIES}

Let $n \in \mathbb{N}$ and $\Omega$ be a bounded open set in $\mathbb{R}^{N}$. By $W^{n, 2}(\Omega)$ we denote the Sobolev space of all functions in $L^{2}(\Omega)$ which admit weak derivatives in $L^{2}(\Omega)$ up to order $n$. By $W_{0}^{n, 2}(\Omega)$ we denote the closure in $W^{n, 2}(\Omega)$ of the space of $C^{\infty}$-functions with compact support in $\Omega$. We consider the weak formulation of problem (1.1). To do so, for any $m \in \mathbb{N}_{0}$ with $0 \leq m \leq n$, we consider the polyharmonic operator $\Delta^{m}$ as the operator from $W_{0}^{n, 2}(\Omega)$ to its dual $\left(W_{0}^{n, 2}(\Omega)\right)^{\prime}$ which takes any $u \in W_{0}^{n, 2}(\Omega)$ to the functional $\Delta^{m}[u]$ defined by

$$
\Delta^{2 s}[u][\varphi]=\int_{\Omega} \Delta^{s} u \Delta^{s} \varphi \mathrm{d} x, \quad \forall \varphi \in W_{0}^{n, 2}(\Omega),
$$

if $m=2 s$ and

$$
\Delta^{2 s+1}[u][\varphi]=-\int_{\Omega} \nabla\left(\Delta^{s} u\right) \cdot \nabla\left(\Delta^{s} \varphi\right) \mathrm{d} x, \quad \forall \varphi \in W_{0}^{n, 2}(\Omega),
$$

if $m=2 s+1$, where $s \in \mathbb{N}_{0}$. Thus, the weak formulation of the classic problem (1.1) reads

$$
(-\Delta)^{n}[u][\varphi]=\lambda(-\Delta)^{m}[u][\varphi], \quad \forall \varphi \in W_{0}^{n, 2}(\Omega) .
$$

By the Poincaré inequality, it follows that the quadratic form defined by $(-\Delta)^{n}[u][u]$ for all $u \in W_{0}^{n, 2}(\Omega)$ is coercive in $W_{0}^{n, 2}(\Omega)$, hence the operator $(-\Delta)^{n}$ is a linear homeomorphism from $W_{0}^{n, 2}(\Omega)$ onto $\left(W_{0}^{n, 2}(\Omega)\right)^{\prime}$. Thus the equation (2.3) is equivalent to the equation $(-\Delta)^{-n} \circ(-\Delta)^{m}[u]=\lambda^{-1} u$, where $(-\Delta)^{-n}$ denotes the inverse of $(-\Delta)^{n}$. It is convenient to endow the space $W_{0}^{n, 2}(\Omega)$ with the scalar product defined by

$$
\left\langle u_{1}, u_{2}\right\rangle_{n}=(-\Delta)^{n}\left[u_{1}\right]\left[u_{2}\right],
$$

for all $u_{1}, u_{2} \in W_{0}^{n, 2}(\Omega)$. The norm induced by this scalar product is equivalent to the standard Sobolev norm. In the sequel, unless otherwise indicated, we shall think of $W_{0}^{n, 2}(\Omega)$ as a Hilbert space equipped with the scalar product (2.4). This allows to give a straightforward proof of the following.

Lemma 2.1. Let $\Omega$ be a bounded open set in $\mathbb{R}^{N}$ and $m, n \in \mathbb{N}_{0}$ with $0 \leq m<n$. The operator $S_{\Omega} \equiv$ $(-\Delta)^{-n} \circ(-\Delta)^{m}$ is a non-negative self-adjoint compact operator in the Hilbert space $W_{0}^{n, 2}(\Omega)$. The spectrum of $S_{\Omega}$ is discrete and consists of a decreasing sequence of positive eigenvalues of finite multiplicity converging to zero. Moreover, the equation $S_{\Omega} u=\mu u$ is satisfied for some $u \in W_{0}^{n, 2}(\Omega), \mu>0$ if and only if equation (2.3) is satisfied with $\lambda=\mu^{-1}$.

Proof. The equality $\left\langle S_{\Omega} u_{1}, u_{2}\right\rangle_{n}=(-\Delta)^{m}\left[u_{1}\right]\left[u_{2}\right]$, for all $u_{1}, u_{2} \in W_{0}^{n, 2}(\Omega)$ and the symmetry of the operator $(-\Delta)^{m}$ implies that $S_{\Omega}$ is a self-adjoint operator. Since $\Omega$ is bounded and $m<n$, the space $W_{0}^{n, 2}(\Omega)$ is compactly embedded into $W_{0}^{m, 2}(\Omega)$. This implies that the operator $(-\Delta)^{m}$ is a compact operator from the space $W_{0}^{n, 2}(\Omega)$ to its dual. The rest of the proof is trivial.

By Lemma 2.1 and standard spectral theory we deduce the following. 
Corollary 2.2. Let $\Omega$ be a bounded open set in $\mathbb{R}^{N}$ and $m, n \in \mathbb{N}_{0}$ with $0 \leq m<n$. The eigenvalues of problem (2.3) are positive, have finite multiplicity and can be represented as a non-decreasing divergent sequence $\lambda_{j}[\Omega], j \in \mathbb{N}$ where each eigenvalue is repeated according to its multiplicity. Moreover,

$$
\lambda_{j}[\Omega] \equiv \lambda_{j}^{n, m}[\Omega]=\min _{\substack{E \subset W_{0}^{n, 2}(\Omega) \\ \operatorname{dim} E=j}} \max _{\substack{u \in E \\ u \neq 0}} R_{\mathrm{nm}}[u],
$$

for all $j \in \mathbb{N}$, where $R_{\mathrm{nm}}[u]$ is the Rayleigh quotient defined by

$$
R_{\mathrm{nm}}[u]=\left\{\begin{array}{lll}
\frac{\int_{\Omega}\left|\Delta^{r} u\right|^{2} \mathrm{~d} x}{\int_{\Omega}\left|\Delta^{s} u\right|^{2} \mathrm{~d} x}, & \text { if } n=2 r, & m=2 s, \\
\frac{\int_{\Omega}\left|\Delta^{r} u\right|^{2} \mathrm{~d} x}{\int_{\Omega}\left|\nabla \Delta^{s} u\right|^{2} \mathrm{~d} x}, & \text { if } n=2 r, & m=2 s+1, \\
\frac{\int_{\Omega}\left|\nabla \Delta^{r} u\right|^{2} \mathrm{~d} x}{\int_{\Omega}\left|\Delta^{s} u\right|^{2} \mathrm{~d} x}, & \text { if } n=2 r+1, & m=2 s, \\
\frac{\int_{\Omega}\left|\nabla \Delta^{r} u\right|^{2} \mathrm{~d} x}{\int_{\Omega}\left|\nabla \Delta^{s} u\right|^{2} \mathrm{~d} x}, & \text { if } n=2 r+1, & m=2 s+1 .
\end{array}\right.
$$

Clearly, the eigenvalues $\lambda_{j}^{n, m}[\Omega]$ depend on $n, m$. However, for the sake of simplicity, we shall write $\lambda_{j}[\Omega]$ instead of $\lambda_{j}^{n, m}[\Omega]$.

\section{Analyticity Results}

Let $\Omega$ be a bounded open set in $\mathbb{R}^{N}$ of class $C^{1}$. In the sequel, we shall consider problem (2.3) in a family of open sets parameterized by suitable diffeomorphisms $\phi$ defined on $\Omega$. Namely, we set

$$
\mathcal{A}_{\Omega}^{n}=\left\{\phi \in C_{b}^{n}\left(\Omega ; \mathbb{R}^{N}\right): \inf _{\substack{x_{1}, x_{2} \in \Omega \\ x_{1} \neq x_{2}}} \frac{\left|\phi\left(x_{1}\right)-\phi\left(x_{2}\right)\right|}{\left|x_{1}-x_{2}\right|}>0\right\},
$$

where $C_{b}^{n}\left(\Omega ; \mathbb{R}^{N}\right)$ denotes the space of all functions from $\Omega$ to $\mathbb{R}^{N}$ of class $C^{n}$, with bounded derivatives up to order $n$. Note that if $\phi \in \mathcal{A}_{\Omega}^{n}$ then $\phi$ is injective, Lipschitz continuous and $\inf _{\Omega}|\operatorname{det} \nabla \phi|>0$. Moreover, $\phi(\Omega)$ is a bounded open set and the inverse map $\phi^{(-1)}$ belongs to $\mathcal{A}_{\phi(\Omega)}^{n}$. Thus it is natural to consider problem $(2.3)$ on $\phi(\Omega)$ and study the dependence of $\lambda_{j}[\phi(\Omega)]$ on $\phi \in \mathcal{A}_{\Omega}^{n}$. To do so, we endow the space $C_{b}^{n}\left(\Omega ; \mathbb{R}^{N}\right)$ with its usual norm $\|f\|_{C_{b}^{n}\left(\Omega ; \mathbb{R}^{N}\right)}=\sup _{|\alpha| \leq n, x \in \Omega}\left|D^{\alpha} f(x)\right|$. Note that $\mathcal{A}_{\Omega}^{n}$ is an open set in $C_{b}^{n}\left(\Omega ; \mathbb{R}^{N}\right)$, see [20], Lemma 3.11. Thus, it makes sense to study differentiability and analyticity properties of the maps $\phi \mapsto \lambda_{j}[\phi(\Omega)]$ defined for $\phi \in \mathcal{A}_{\Omega}^{n}$. For simplicity, we write $\lambda_{j}[\phi]$ instead of $\lambda_{j}[\phi(\Omega)]$. As in [20], we fix a finite set of indeces $F \subset \mathbb{N}$ and we consider those maps $\phi \in \mathcal{A}_{\Omega}^{n}$ for which the eigenvalues with indeces in $F$ do not coincide with eigenvalues with indeces not in $F$; namely we set

$$
\mathcal{A}_{F, \Omega}^{n, m}=\left\{\phi \in \mathcal{A}_{\Omega}^{n}: \lambda_{j}[\phi] \neq \lambda_{l}[\phi], \forall j \in F, l \in \mathbb{N} \backslash F\right\} .
$$

It is also convenient to consider those maps $\phi \in \mathcal{A}_{F, \Omega}^{n, m}$ such that all the eigenvalues with index in $F$ coincide and set

$$
\Theta_{F, \Omega}^{n, m}=\left\{\phi \in \mathcal{A}_{F, \Omega}^{n, m}: \lambda_{j_{1}}[\phi]=\lambda_{j_{2}}[\phi], \forall j_{1}, j_{2} \in F\right\} .
$$

For $\phi \in \mathcal{A}_{F, \Omega}^{n, m}$, the elementary symmetric functions of the eigenvalues with index in $F$ are defined by

$$
\Lambda_{F, h}[\phi]=\sum_{\substack{j_{1}, \ldots, j_{h} \in F \\ j_{1}<\cdots<j_{h}}} \lambda_{j_{1}}[\phi] \cdots \lambda_{j_{h}}[\phi], \quad h=1, \ldots,|F| .
$$

The main result of this section is the following generalization to polyharmonic operators on smooth domains of the results in [20], Section 3 concerning the Dirichlet Laplacian on rough domains. 
Theorem 3.1. Let $\Omega$ be a bounded open set in $\mathbb{R}^{N}$ of class $C^{1}, n, m \in \mathbb{N}_{0}$ with $0 \leq m<n$, and $F$ be a finite set in $\mathbb{N}$. The set $\mathcal{A}_{F, \Omega}^{n, m}$ is open in $C_{b}^{n}\left(\Omega ; \mathbb{R}^{N}\right)$ and the real-valued maps which take $\phi \in \mathcal{A}_{F, \Omega}^{n, m}$ to $\Lambda_{F, h}[\phi]$ are real-analytic on $\mathcal{A}_{F, \Omega}^{n, m}$ for all $h=1, \ldots,|F|$. Moreover, if $\tilde{\phi} \in \Theta_{F, \Omega}^{n, m}$ is such that the eigenvalues $\lambda_{j}[\tilde{\phi}]$ assume the common value $\lambda_{F}[\tilde{\phi}]$ for all $j \in F$, and $\tilde{\phi}(\Omega)$ is of class $C^{2 n}$ then the Frechét differential of the map $\Lambda_{F, h}$ at the point $\tilde{\phi}$ is delivered by the formula

$$
\left.d\right|_{\phi=\tilde{\phi}} \Lambda_{F, h}[\psi]=-\lambda_{F}^{h}[\tilde{\phi}]\left(\begin{array}{c}
|F|-1 \\
h-1
\end{array}\right) \sum_{l=1}^{|F|} \int_{\partial \tilde{\phi}(\Omega)}\left(\frac{\partial^{n} v_{l}}{\partial \nu^{n}}\right)^{2}\left(\psi \circ \tilde{\phi}^{(-1)}\right) \cdot \nu \mathrm{d} \sigma,
$$

for all $\psi \in C_{b}^{n}\left(\Omega ; \mathbb{R}^{N}\right)$, where $\left\{v_{l}\right\}_{l \in F}$ is an orthonormal basis in $W_{0}^{n, 2}(\tilde{\phi}(\Omega))$ (with respect to the scalar product (2.4)) of the eigenspace associated with $\lambda_{F}[\tilde{\phi}]$, and $\nu$ denotes the unit outer normal to $\partial \tilde{\phi}(\Omega)$.

Note that formula (3.2) is a generalization of the celebrated Hadamard formula, see Grinfeld [12] for a recent paper on this topic; see also Ortega and Zuazua [24] for the analysis of associated bifurcation phenomena concerning multiple eigenvalues of the biharmonic operator subject to Dirichlet boundary conditions.

In order to prove Theorem 3.1 we consider equation $(2.3)$ on $\phi(\Omega)$ and pull it back to $\Omega$. Namely, we consider the equation

$$
(-\Delta)^{n}[v][\psi]=\lambda(-\Delta)^{m}[v][\psi], \quad \forall \psi \in W_{0}^{n, 2}(\phi(\Omega)),
$$

in the unknowns $\left.v \in W_{0}^{n, 2}(\phi(\Omega)), \lambda \in\right] 0, \infty[$. Recall that the pull-back to $\Omega$ of the classic Laplace operator on $\phi(\Omega)$ is defined by

$$
\Delta_{\phi} u=\left(\Delta\left(u \circ \phi^{(-1)}\right)\right) \circ \phi
$$

for all $u \in W_{l o c}^{2,1}(\Omega), \phi \in \mathcal{A}_{\Omega}^{2}$. The operator $\Delta_{\phi}$ is in fact the Laplace-Beltrami operator associated with the change of variables defined by $\phi$. Note that

$$
\Delta_{\phi}^{s} u=\left(\Delta^{s}\left(u \circ \phi^{(-1)}\right)\right) \circ \phi
$$

for all $u \in W_{l o c}^{2 s, 1}(\Omega), \phi \in \mathcal{A}_{\Omega}^{2 s}$. For any $0 \leq m \leq n$, the operator $\Delta_{\phi}^{m}$ can be considered as the operator acting from $W_{0}^{n, 2}(\Omega)$ to its dual, which takes any $u \in W_{0}^{n, 2}(\Omega)$ to the functional $\Delta_{\phi}^{n}[u]$ defined by

$$
\Delta_{\phi}^{m}[u][\varphi]=\Delta^{m}\left[u \circ \phi^{(-1)}\right]\left[\varphi \circ \phi^{(-1)}\right],
$$

for all $\varphi \in W_{0}^{n, 2}(\Omega)$. More precisely, if $m=2 s, s \in \mathbb{N}_{0}$ then

$$
\Delta_{\phi}^{2 s}[u][\varphi]=\int_{\Omega} \Delta_{\phi}^{s} u \Delta_{\phi}^{s} \varphi|\operatorname{det} \nabla \phi| \mathrm{d} x,
$$

for all $\varphi \in W_{0}^{n, 2}(\Omega)$. If $m=2 s+1, s \in \mathbb{N}_{0}$ then

$$
-\Delta_{\phi}^{2 s+1}[u][\varphi]=\int_{\Omega} \nabla\left(\Delta_{\phi}^{s} u\right)(\nabla \phi)^{-1}(\nabla \phi)^{-t} \nabla^{t}\left(\Delta_{\phi}^{s} \varphi\right)|\operatorname{det} \nabla \phi| \mathrm{d} x
$$

for all $\varphi \in W_{0}^{n, 2}(\Omega)$, where $(\nabla \phi)^{-1}$ denotes the inverse of the Jacobian matrix of $\phi$ and $(\nabla \phi)^{-t}$ the transpose of $(\nabla \phi)^{-1}$. Note that the map from $W_{0}^{n, 2}(\Omega)$ to $W_{0}^{n, 2}(\phi(\Omega))$ which maps $u$ to $u \circ \phi^{(-1)}$ for all $u \in W_{0}^{n, 2}(\Omega)$ is a linear homeomorphism. Hence, equation (3.3) is equivalent to

$$
\left(-\Delta_{\phi}\right)^{n}[u][\varphi]=\lambda\left(-\Delta_{\phi}\right)^{m}[u][\varphi], \quad \forall \varphi \in W_{0}^{n, 2}(\Omega)
$$

where $u=v \circ \phi$. It is also natural to pull-back the scalar product of $W_{0}^{n, 2}(\phi(\Omega))$ to $\Omega$ by setting

$$
\left\langle u_{1}, u_{2}\right\rangle_{n, \phi}=\left\langle u_{1} \circ \phi^{(-1)}, u_{2} \circ \phi^{(-1)}\right\rangle_{n}
$$


for all $u_{1}, u_{2} \in W_{0}^{n, 2}(\Omega)$, where $\langle\cdot, \cdot\rangle_{n}$ is the scalar product in $W_{0}^{n, 2}(\phi(\Omega))$ defined by $(2.4)$. By $W_{0, \phi}^{n, 2}(\Omega)$ we denote the Hilbert space $W_{0}^{n, 2}(\Omega)$ endowed with the scalar product $\langle\cdot, \cdot\rangle_{n, \phi}$. It turns out that the operator $S_{\phi(\Omega)}$ defined in Lemma 2.1 is unitarily equivalent to the operator $T_{\phi}$ defined on $W_{0, \phi}^{n, 2}(\Omega)$ by

$$
T_{\phi}=\left(-\Delta_{\phi}\right)^{-n} \circ\left(-\Delta_{\phi}\right)^{m} \text {. }
$$

Thus we can prove the following lemma where $\mathcal{L}\left(W_{0}^{n, 2}(\Omega)\right)$ denotes the space of linear bounded operators from $W_{0}^{n, 2}(\Omega)$ to itself and and $\mathcal{B}_{s}\left(W_{0}^{n, 2}(\Omega)\right.$ ) denotes the space of bilinear forms on $W_{0}^{n, 2}(\Omega)$ (both spaces are equipped with their usual norms).

Lemma 3.2. Let $\Omega$ be a bounded open set in $\mathbb{R}^{N}$ of class $C^{1}, n, m \in \mathbb{N}_{0}, 0 \leq m<n$. The operator $T_{\phi}$ defined in (3.10) is non-negative self-adjoint and compact on the Hilbert space $W_{0, \phi}^{n, 2}(\Omega)$. The equation (3.3) is satisfied for some $v \in W_{0}^{n, 2}(\phi(\Omega))$ if and only if the equation $T_{\phi} u=\mu u$ is satisfied with $u=v \circ \phi$ and $\mu=\lambda^{-1}$. Moreover, the map from $\mathcal{A}_{\Omega}^{n}$ to $\mathcal{L}\left(W_{0}^{n, 2}(\Omega)\right) \times \mathcal{B}_{s}\left(W_{0}^{n, 2}(\Omega)\right)$ which takes $\phi \in \mathcal{A}_{\Omega}^{n}$ to $\left(T_{\phi},\langle\cdot, \cdot\rangle_{n, \phi}\right)$ is real-analytic.

Proof. Since the operator $T_{\phi}$ is unitarily equivalent to the operator $S_{\phi(\Omega)}$, the first part of the lemma immediately follows by Lemma 2.1. In order to prove the real-analytic dependence of $T_{\phi}$ upon $\phi$, we note that by standard calculus

$$
\Delta_{\phi} u=\sum_{r, s, i=1}^{N}\left(\frac{\partial^{2} u}{\partial x_{r} \partial x_{s}} \sigma_{r i} \sigma_{s i}+\frac{\partial u}{\partial x_{r}} \frac{\partial \sigma_{r i}}{\partial x_{s}} \sigma_{s i}\right)
$$

for all $u \in W^{2,2}(\Omega)$, where $\sigma=(\nabla \phi)^{-1}$ (see also [20], Prop. 3.1). By formula (3.11), it follows that the map from $\mathcal{A}_{\Omega}^{n} \times W^{n, 2}(\Omega)$ to $W^{n-2,2}(\Omega)$ which takes $(\phi, u)$ to $\Delta_{\phi} u$ is real-analytic. Thus also the maps from $\mathcal{A}_{\Omega}^{n} \times W^{n, 2}(\Omega)$ to $L^{2}(\Omega)$ which take $(\phi, u)$ to $\Delta_{\phi}^{s} u$ for all $s \in \mathbb{N}_{0}$ with $0 \leq s \leq n / 2$, are real-analytic since they are compositions of real-analytic maps. This, combined with formulas (3.6) and (3.7), implies the real-analytic dependence of $T_{\phi}$ and $\langle\cdot, \cdot\rangle_{n, \phi}$ upon $\phi$.

Proof of Theorem 3.1. We denote by $\mu_{j}[\phi], j \in \mathbb{N}$, the eigenvalues of $T_{\phi}$. By Lemma $3.2, \mu_{j}[\phi]=\lambda_{j}^{-1}[\phi]$ for all $j \in \mathbb{N}$, hence the set $\mathcal{A}_{F, \Omega}^{n, m}$ coincides with the set $\left\{\phi \in \mathcal{A}_{\Omega}^{n}: \mu_{j}[\phi] \neq \mu_{l}[\phi], \forall j \in F, l \in \mathbb{N} \backslash F\right\}$. By Lemma 3.2, $T_{\phi}$ is self-adjoint with respect to the scalar product $\langle\cdot, \cdot\rangle_{n, \phi}$ and both $T_{\phi}$ and $\langle\cdot, \cdot\rangle_{n, \phi}$ depend real-analytically on $\phi$. Thus, by applying [20], Theorem 2.30 , it follows that $\mathcal{A}_{F, \Omega}^{n, m}$ is an open set in $C_{b}^{n}\left(\Omega ; \mathbb{R}^{N}\right)$ and the functions which take $\phi \in \mathcal{A}_{F, \Omega}^{n, m}$ to

$$
\Gamma_{F, h}[\phi]=\sum_{\substack{j_{1}, \ldots, j_{h} \in F \\ j_{1}<\cdots<j_{h}}} \mu_{j_{1}}[\phi] \cdots \mu_{j_{h}}[\phi]
$$

are real-analytic for all $h=1, \ldots,|F|$. Since

$$
\Lambda_{F, h}[\phi]=\frac{\Gamma_{F,|F|-h}[\phi]}{\Gamma_{F,|F|}[\phi]},
$$

for all $h=1, \ldots,|F|$, where $\Gamma_{F, 0}[\phi]=1$, it follows that $\Lambda_{F, h}[\phi]$ depends real-analytically on $\phi \in \mathcal{A}_{F, \Omega}^{n, m}$.

It remains to prove formula (3.2). Let $\tilde{\phi} \in \Theta_{F, \Omega}^{n, m}, \lambda_{F}[\tilde{\phi}]$ and $\left\{v_{l}\right\}_{l \in F}$ be as in the statement. We set $u_{l}=v_{l} \circ \tilde{\phi}$ for all $l \in F$ and we note that $\left\{u_{l}\right\}_{l \in F}$ is an orthonormal basis in $W_{0, \tilde{\phi}}^{n, 2}(\Omega)$ for the eigenspace corresponding to the eigenvalue $\lambda_{F}^{-1}[\tilde{\phi}]$ of the operator $T_{\tilde{\phi}}$. By [20], Theorem 2.30, it follows that

$$
\left.\mathrm{d}\right|_{\phi=\tilde{\phi}} \Gamma_{F, h}[\psi]=\lambda_{F}^{1-h}[\tilde{\phi}]\left(\begin{array}{c}
|F|-1 \\
h-1
\end{array}\right) \sum_{l \in F}\left\langle\left.\mathrm{~d}\right|_{\phi=\tilde{\phi}} T_{\phi}[\psi]\left[u_{l}\right], u_{l}\right\rangle_{n, \tilde{\phi}}
$$

for all $\psi \in C_{b}^{n}\left(\Omega ; \mathbb{R}^{N}\right)$. Note that by standard regularity theory (see e.g., Agmon [1], Thm. 9.8) $v_{l} \in W^{2 n, 2}(\tilde{\phi}(\Omega)$ ) for all $l \in F$. 
By standard calculus, equalities (2.4), (3.9), Theorem 5.2, by observing that $\frac{\partial^{m} v_{l}}{\partial \nu^{m}}=0$ on $\partial \tilde{\phi}(\Omega)$ and $(-\Delta)^{n} v_{l}=\lambda_{F}[\tilde{\phi}](-\Delta)^{m} v_{l}$, we have

$$
\begin{aligned}
\left\langle\left.\mathrm{d}\right|_{\phi=\tilde{\phi}} T_{\phi}[\psi]\left[u_{l}\right], u_{l}\right\rangle_{n, \tilde{\phi}} \\
=\left(\left.\mathrm{d}\right|_{\phi=\tilde{\phi}}\left(-\Delta_{\phi}\right)^{m}[\psi]\right)\left[u_{l}\right]\left[u_{l}\right]-\lambda_{F}^{-1}[\tilde{\phi}]\left(\left.\mathrm{d}\right|_{\phi=\tilde{\phi}}\left(-\Delta_{\phi}\right)^{n}[\psi]\right)\left[u_{l}\right]\left[u_{l}\right] \\
=-\int_{\partial \tilde{\phi}(\Omega)}\left(\frac{\partial^{m} v_{l}}{\partial \nu^{m}}\right)^{2} \zeta \cdot \nu \mathrm{d} \sigma-2 \int_{\partial \tilde{\phi}(\Omega)}(-\Delta)^{m} v_{l} \nabla v_{l} \cdot \zeta \mathrm{d} \sigma \\
\quad+\lambda_{F}^{-1}[\tilde{\phi}] \int_{\partial \tilde{\phi}(\Omega)}\left(\frac{\partial^{n} v_{l}}{\partial \nu^{n}}\right)^{2} \zeta \cdot \nu \mathrm{d} \sigma+2 \lambda_{F}^{-1}[\tilde{\phi}] \int_{\partial \tilde{\phi}(\Omega)}(-\Delta)^{n} v_{l} \nabla v_{l} \cdot \zeta \mathrm{d} \sigma \\
=\lambda_{F}^{-1}[\tilde{\phi}] \int_{\partial \tilde{\phi}(\Omega)}\left(\frac{\partial^{n} v_{l}}{\partial \nu^{n}}\right)^{2} \zeta \cdot \nu \mathrm{d} \sigma,
\end{aligned}
$$

where we have set $\zeta=\psi \circ \tilde{\phi}^{(-1)}$. Formula (3.2) easily follows by combining formulas (3.13)-(3.15).

\section{ISOVOLUMETRIC PERTURBATIONS}

Consider the following extremum problems for the symmetric functions of the eigenvalues

$$
\min _{V[\phi]=\text { const }} \Lambda_{F, h}[\phi] \quad \text { or } \max _{V[\phi]=\text { const }} \Lambda_{F, h}[\phi],
$$

where $V[\phi]$ denotes the $N$-dimensional Lebesgue measure of $\phi(\Omega)$.

Note that if $\tilde{\phi} \in \mathcal{A}_{\Omega}^{n}$ is a minimizer or maximizer in (4.1) then $\tilde{\phi}$ is a critical domain transformation for the map $\phi \mapsto \Lambda_{F, h}[\phi]$ subject to volume constraint, i.e.,

$$
\left.\left.\operatorname{Ker} \mathrm{d}\right|_{\phi=\tilde{\phi}} V \subset \operatorname{Ker} \mathrm{d}\right|_{\phi=\tilde{\phi}} \Lambda_{F, h},
$$

where $V$ is the real valued function defined on $\mathcal{A}_{\Omega}^{n}$ which takes $\phi \in \mathcal{A}_{\Omega}^{n}$ to $V[\phi]$.

The following theorem provides a characterization of all critical domain transformations $\phi$. See [19] for the case of the Dirichlet and Neumann Laplacians.

Theorem 4.1. Let $\Omega$ be a bounded open set in $\mathbb{R}^{N}$ of class $C^{1}, n, m \in \mathbb{N}_{0}$ with $0 \leq m<n$, and $F$ be a finite subset of $\mathbb{N}$. Assume that $\tilde{\phi} \in \Theta_{F, \Omega}^{n, m}$ is such that $\tilde{\phi}(\Omega)$ is of class $C^{2 n}$ and that the eigenvalues $\lambda_{j}[\tilde{\phi}]$ have the common value $\lambda_{F}[\tilde{\phi}]$ for all $j \in F$. Let $\left\{v_{l}\right\}_{l \in F}$ be an orthornormal basis in $W_{0}^{n, 2}(\tilde{\phi}(\Omega))$ of the eigenspace corresponding to $\lambda_{F}[\tilde{\phi}]$. Then $\tilde{\phi}$ is a critical domain transformation for any of the functions $\Lambda_{F, h}, h=1, \ldots,|F|$, with volume constraint if and only if there exists $C \in \mathbb{R}$ such that

$$
\sum_{l \in F}\left|\frac{\partial^{n} v_{l}}{\partial \nu^{n}}\right|^{2}=C, \quad \text { on } \partial \tilde{\phi}(\Omega) .
$$

Proof. Note that $V[\phi]=\int_{\Omega}|\operatorname{det} \nabla \phi| \mathrm{d} x$. Moreover, by standard calculus

$$
\left[\left(\left.\mathrm{d}\right|_{\phi=\tilde{\phi}}(\operatorname{det} \nabla \phi)[\psi]\right) \circ \tilde{\phi}^{(-1)}\right] \operatorname{det} \nabla \tilde{\phi}^{(-1)}=\operatorname{div}\left(\psi \circ \tilde{\phi}^{(-1)}\right),
$$

for all $\psi \in C_{b}^{n}\left(\Omega ; \mathbb{R}^{N}\right)$. Thus by formula (4.4) it follows that

$$
\left.\mathrm{d}\right|_{\phi=\tilde{\phi}} V[\psi]=\int_{\partial \tilde{\phi}(\Omega)}\left(\psi \circ \tilde{\phi}^{(-1)}\right) \cdot \nu \mathrm{d} \sigma
$$


for all $\psi \in C_{b}^{n}\left(\Omega ; \mathbb{R}^{N}\right)$. The proof of (4.3) follows immediately by formulas (3.2) and (4.5), and by observing that condition (4.2) is satisfied if and only if there exists $c \in \mathbb{R}$ (a Lagrange multiplier) such that

$$
\left.\mathrm{d}\right|_{\phi=\tilde{\phi}} \Lambda_{F, h}=\left.c \mathrm{~d}\right|_{\phi=\tilde{\phi}} V .
$$

Finally, we can prove the following.

Theorem 4.2. Let the same assumptions of Theorem 4.1 hold. If $\tilde{\phi}(\Omega)$ is a ball then condition (4.3) is satisfied.

Proof. Without any loss of generality, we assume that $\tilde{\phi}(\Omega)$ is a ball with radius $R$ centered at zero. By the rotation invariance of the Laplace operator, $\left\{v_{l} \circ A\right\}_{l \in F}$ is an orthonormal basis of the eigenspace corresponding to $\lambda_{F}[\tilde{\phi}]$ for all $A \in O_{N}(\mathbb{R})$ where $O_{N}(\mathbb{R})$ denotes the group of orthogonal linear transformations in $\mathbb{R}^{N}$. Since both $\left\{v_{l}\right\}_{l \in F}$ and $\left\{v_{l} \circ A\right\}_{l \in F}$ are orthonormal bases of the same space, it follows that $\sum_{l=1}^{|F|} v_{l}^{2} \circ A=\sum_{l=1}^{|F|} v_{l}^{2}$, for all $A \in O_{n}(\mathbb{R})$. Thus $\sum_{l=1}^{|F|} v_{l}^{2}$ is a radial function. By differentiating with respect to the radial coordinate $r$, by the Leibniz formula and by recalling that all derivatives up to order $n-1$ of the eigenfunctions vanish at the boundary of $\tilde{\phi}(\Omega)$, we obtain that

$$
\left.\frac{\partial^{2 n} v_{l}^{2}}{\partial r^{2 n}}\right|_{r=R}=\left.\sum_{k=0}^{2 n}\left(\begin{array}{c}
2 n \\
k
\end{array}\right)\left(\frac{\partial^{k} v_{l}}{\partial r^{k}} \frac{\partial^{2 n-k} v_{l}}{\partial r^{2 n-k}}\right)\right|_{r=R}=\left.\left(\begin{array}{c}
2 n \\
n
\end{array}\right)\left(\frac{\partial^{n} v_{l}}{\partial r^{n}}\right)^{2}\right|_{r=R} .
$$

Since $\sum_{l \in F} \frac{\partial^{2 n} v_{l}^{2}}{\partial r^{2 n}}$ is a radial function, then by formula (4.7) we conclude that the $\sum_{l \in F}\left(\frac{\partial^{n} v_{l}}{\partial \nu^{n}}\right)^{2}$ is constant on $\partial \tilde{\phi}(\Omega)$.

It would be interesting to clarify whether balls are local minimizers or maximizers for the eigenvalues or their symmetric functions. With regard to this, we mention that it is proved in Wolf and Keller [29], Theorem 8.3 that the disk in the plane is a local minimizer for the third eigenvalue of the Dirichlet Laplacian.

\section{A formula for the Frechét differential of the "Poly-Laplace-Beltrami" OPERATOR}

In this section we prove Theorem 5.2 which has its own interest since it provides an explicit formula for the Frechét differential with respect to $\phi$ of the weak "poly-Laplace-Beltrami" operator $\Delta_{\phi}^{n}$ defined by (3.6), (3.7). That formula has been used in the proof of (3.2).

Lemma 5.1. Let $\Omega$ be a bounded open set in $\mathbb{R}^{N}$ of class $C^{1}, s \in \mathbb{N}, u_{1} \in L^{2}(\Omega), u_{2} \in W_{0}^{2 s, 2}(\Omega)$. Let $\tilde{\phi} \in \mathcal{A}_{\Omega}^{2 s}$ and $v_{i}=u_{i} \circ \tilde{\phi}^{(-1)}, i=1,2$. Assume that $\tilde{\phi}(\Omega)$ is of class $C^{1}$ and that $v_{1} \in W^{2 s, 2}(\tilde{\phi}(\Omega)), v_{2} \in W^{2 s+1,2}(\tilde{\phi}(\Omega))$. Then

$$
\begin{aligned}
\left.\int_{\Omega} u_{1} \mathrm{~d}\right|_{\phi=\tilde{\phi}} \Delta_{\phi}^{s}[\psi] u_{2}|\operatorname{det} \nabla \tilde{\phi}| \mathrm{d} x & \\
& =\int_{\tilde{\phi}(\Omega)}\left(v_{1} \nabla \Delta^{s} v_{2}-\Delta^{s} v_{1} \nabla v_{2}\right) \cdot \zeta \mathrm{d} y-\int_{\partial \tilde{\phi}(\Omega)} v_{1} \Delta^{s} v_{2} \zeta \cdot \nu \mathrm{d} \sigma,
\end{aligned}
$$

for all $\psi \in C_{b}^{2 s}\left(\Omega ; \mathbb{R}^{N}\right)$, where $\zeta=\psi \circ \tilde{\phi}^{(-1)}$.

Proof. First, we recall the following formula from [21], Lemma 3.42 which holds for any $u \in W^{2,2}(\Omega)$ :

$$
\left(\left.\mathrm{d}\right|_{\phi=\tilde{\phi}} \Delta_{\phi}[\psi] u\right) \circ \tilde{\phi}^{(-1)}=-2 \sum_{i, j=1}^{N} \frac{\partial^{2}\left(u \circ \tilde{\phi}^{(-1)}\right)}{\partial y_{i} \partial y_{j}} \frac{\partial \zeta_{j}}{\partial y_{i}}-\sum_{j=1}^{N} \frac{\partial\left(u \circ \tilde{\phi}^{(-1)}\right)}{\partial y_{j}} \Delta \zeta_{j} .
$$


We observe that

$$
\left.d\right|_{\phi=\tilde{\phi}} \Delta_{\phi}^{s}[\psi]=\sum_{\substack{h, k=0 \\ h+k=s-1}}^{s-1} \Delta_{\tilde{\phi}}^{h} \circ\left(\left.d\right|_{\phi=\tilde{\phi}} \Delta_{\phi}[\psi]\right) \circ \Delta_{\tilde{\phi}}^{k},
$$

By formulas (5.2) and (5.3), by changing variables in integrals and integrating by parts, we obtain

$$
\begin{aligned}
\left.\int_{\Omega} u_{1} \mathrm{~d}\right|_{\phi=\tilde{\phi}} \Delta_{\phi}^{s}[\psi] u_{2}|\operatorname{det} \nabla \tilde{\phi}| \mathrm{d} x \\
=-\sum_{\substack{h, k=0 \\
h+k=s-1}}^{s-1} \int_{\tilde{\phi}(\Omega)} \Delta^{h} v_{1}\left(2 \sum_{i, j=1}^{N} \frac{\partial^{2} \Delta^{k} v_{2}}{\partial y_{i} \partial y_{j}} \frac{\partial \zeta_{j}}{\partial y_{i}}+\sum_{j=1}^{N} \frac{\partial \Delta^{k} v_{2}}{\partial y_{j}} \Delta \zeta_{j}\right) \mathrm{d} y \\
=\sum_{\substack{h, k=0 \\
h+k=s-1}}^{s-1} \int_{\tilde{\phi}(\Omega)} \sum_{i, j=1}^{N}\left[\frac{\partial \Delta^{h} v_{1}}{\partial y_{i}} \frac{\partial \Delta^{k} v_{2}}{\partial y_{j}}\left(\frac{\partial \zeta_{j}}{\partial y_{i}}+\frac{\partial \zeta_{i}}{\partial y_{j}}\right)\right] \\
\quad-\left(\Delta^{h} v_{1} \Delta^{k+1} v_{2}+\nabla \Delta^{h} v_{1} \nabla \Delta^{k} v_{2}\right) \operatorname{div} \zeta \mathrm{d} y
\end{aligned}
$$

see also [21], Formula (3.45). Moreover, integrating by parts yields

$$
\begin{aligned}
\int_{\tilde{\phi}(\Omega)} & \frac{\partial \Delta^{h} v_{1}}{\partial y_{i}} \frac{\partial \Delta^{k} v_{2}}{\partial y_{j}}\left(\frac{\partial \zeta_{j}}{\partial y_{i}}+\frac{\partial \zeta_{i}}{\partial y_{j}}\right) \mathrm{d} y=-\int_{\partial \tilde{\phi}(\Omega)} \Delta^{h} v_{1} \Delta^{k+1} v_{2} \zeta \cdot \nu \mathrm{d} \sigma \\
& +\int_{\tilde{\phi}(\Omega)} \Delta^{h} v_{1} \nabla \Delta^{k+1} v_{2} \cdot \zeta \mathrm{d} y+\int_{\tilde{\phi}(\Omega)} \Delta^{h} v_{1} \Delta^{k+1} v_{2} \operatorname{div} \zeta \mathrm{d} y \\
& +\int_{\tilde{\phi}(\Omega)} \nabla \Delta^{h} v_{1} \cdot \nabla \Delta^{k} v_{2} \operatorname{div} \zeta \mathrm{d} y-\int_{\tilde{\phi}(\Omega)} \Delta^{h+1} v_{1} \nabla \Delta^{k} v_{2} \cdot \zeta \mathrm{d} y .
\end{aligned}
$$

By observing that the first summand in the right-hand side of (5.5) vanishes if $k<s-1$, and by combining (5.4) and (5.5), we obtain a telescopic sum and we deduce the validity of (5.1).

Theorem 5.2. Let $\Omega$ be a bounded open set in $\mathbb{R}^{N}$ of class $C^{1}, n \in \mathbb{N}, u_{1}, u_{2} \in W_{0}^{n, 2}(\Omega)$. Let $\tilde{\phi} \in \mathcal{A}_{\Omega}^{n}$ and $v_{i}=u_{i} \circ \tilde{\phi}^{(-1)}, i=1,2$. Assume that $\tilde{\phi}(\Omega)$ is of class $C^{1}$ and that $v_{1}, v_{2} \in W^{2 n, 2}(\tilde{\phi}(\Omega))$. Then

$$
\begin{aligned}
\left(\left.\mathrm{d}\right|_{\phi=\tilde{\phi}}\left(-\Delta_{\phi}\right)^{n}[\psi]\right)\left[u_{1}\right]\left[u_{2}\right]= & -\int_{\partial \tilde{\phi}(\Omega)} \frac{\partial^{n} v_{1}}{\partial \nu^{n}} \frac{\partial^{n} v_{2}}{\partial \nu^{n}} \zeta \cdot \nu \mathrm{d} \sigma \\
& -\int_{\tilde{\phi}(\Omega)}\left((-\Delta)^{n} v_{1} \nabla v_{2}+(-\Delta)^{n} v_{2} \nabla v_{1}\right) \cdot \zeta \mathrm{d} y,
\end{aligned}
$$

for all $\psi \in C_{b}^{n}\left(\Omega ; \mathbb{R}^{N}\right)$, where $\zeta=\psi \circ \tilde{\phi}^{(-1)}$.

Proof. First, we consider the case where $n$ is an even number of the form $n=2 s$ with $s \in \mathbb{N}_{0}$. By formula (3.6) and standard calculus we have

$$
\begin{aligned}
\left(\left.d\right|_{\phi=\tilde{\phi}} \Delta_{\phi}^{2 s}[\psi]\left(u_{1}\right)\right)\left(u_{2}\right)= & \left.\int_{\Omega} d\right|_{\phi=\tilde{\phi}} \Delta_{\phi}^{s} u_{1}[\psi] \Delta_{\tilde{\phi}}^{s} u_{2}|\operatorname{det} D \tilde{\phi}| \mathrm{d} x \\
& +\left.\int_{\Omega} \Delta_{\tilde{\phi}}^{s} u_{1} d\right|_{\phi=\tilde{\phi}} \Delta_{\phi}^{s} u_{2}[\psi]|\operatorname{det} D \tilde{\phi}| \mathrm{d} x \\
& +\left.\int_{\Omega} \Delta_{\tilde{\phi}}^{s} u_{1} \Delta_{\tilde{\phi}}^{s} u_{2} d\right|_{\phi=\tilde{\phi}}|\operatorname{det} D \phi|[\psi] \mathrm{d} x
\end{aligned}
$$


By formula (4.4)

$$
\left.\int_{\Omega} \Delta_{\tilde{\phi}}^{s} u_{1} \Delta_{\tilde{\phi}}^{s} u_{2} d\right|_{\phi=\tilde{\phi}}|\operatorname{det} D \phi|[\psi] \mathrm{d} x=\int_{\tilde{\phi}(\Omega)} \Delta^{s} \tilde{v}_{1} \Delta^{s} \tilde{v}_{2} \operatorname{div} \zeta \mathrm{d} y
$$

Formula (5.6) easily follows by combining formulas (5.1), (5.7), (5.8), by integrating by parts and by observing that $\Delta^{s} v_{i}=\frac{\partial^{2 s} v_{i}}{\partial \nu^{2 s}}$ on $\partial \tilde{\phi}(\Omega)$ since $v_{i} \in W_{0}^{2 s, 2}(\tilde{\phi}(\Omega))$.

Now, we consider the case where $n$ is an odd number of the form $n=2 s+1$ with $s \in \mathbb{N}_{0}$. By formula (3.7) and standard calculus we have

$$
\begin{array}{r}
\left(\left.d\right|_{\phi=\tilde{\phi}} \Delta_{\phi}^{2 s+1}[\psi]\left(u_{1}\right)\right)\left(u_{2}\right)=\int_{\tilde{\phi}(\Omega)} \nabla \Delta^{s} v_{1}\left(\nabla \zeta+\nabla^{t} \zeta\right) \nabla^{t} \Delta^{s} v_{2} \mathrm{~d} y \\
-\int_{\tilde{\phi}(\Omega)} \nabla \Delta^{s} v_{1} \nabla \Delta^{s} v_{2} \operatorname{div} \zeta \mathrm{d} y-\int_{\tilde{\phi}(\Omega)} \nabla \Delta^{s} v_{1} \nabla\left(\left(\left.d\right|_{\phi=\tilde{\phi}} \Delta_{\phi}^{s} u_{2}[\psi]\right) \circ \tilde{\phi}^{(-1)}\right) \mathrm{d} y \\
-\int_{\tilde{\phi}(\Omega)} \nabla\left(\left(\left.d\right|_{\phi=\tilde{\phi}} \Delta_{\phi}^{s} u_{1}[\psi]\right) \circ \tilde{\phi}^{(-1)}\right) \nabla \Delta^{s} v_{2} \mathrm{~d} y .
\end{array}
$$

Moreover, integrating by parts yields

$$
\begin{aligned}
\int_{\tilde{\phi}(\Omega)} \nabla & \Delta^{s} v_{1}\left(\nabla \zeta+\nabla^{t} \zeta\right) \nabla^{t} \Delta^{s} v_{2} \mathrm{~d} y \\
= & \sum_{h, k=1}^{N} \int_{\tilde{\phi}(\Omega)}\left(\frac{\partial \zeta_{h}}{\partial y_{k}} \frac{\partial \Delta^{s} v_{1}}{\partial y_{h}} \frac{\partial \Delta^{s} v_{2}}{\partial y_{k}}+\frac{\partial \zeta_{k}}{\partial y_{h}} \frac{\partial \Delta^{s} v_{1}}{\partial y_{h}} \frac{\partial \Delta^{s} v_{2}}{\partial y_{k}}\right) \mathrm{d} x \\
= & 2 \int_{\partial \tilde{\phi}(\Omega)} \frac{\partial \Delta^{s} v_{1}}{\partial \nu} \frac{\partial \Delta^{s} v_{2}}{\partial \nu} \zeta \cdot \nu \mathrm{d} \sigma \\
& -\sum_{h, k=1}^{N} \int_{\tilde{\phi}(\Omega)}\left(\frac{\partial^{2} \Delta^{s} v_{1}}{\partial y_{h} \partial y_{k}} \frac{\partial \Delta^{s} v_{2}}{\partial y_{k}} \zeta_{h}+\frac{\partial \Delta^{s} v_{1}}{\partial y_{h}} \frac{\partial^{2} \Delta^{s} v_{2}}{y_{k}^{2}} \zeta_{h}\right) \mathrm{d} y \\
& -\sum_{h, k=1}^{N} \int_{\tilde{\phi}(\Omega)}\left(\frac{\partial^{2} \Delta^{s} v_{1}}{\partial y_{h}^{2}} \frac{\partial \Delta^{s} v_{2}}{\partial y_{k}} \zeta_{k}+\frac{\partial \Delta^{s} v_{1}}{\partial y_{h}} \frac{\partial^{2} \Delta^{s} v_{2}}{\partial y_{h} \partial y_{k}} \zeta_{k}\right) \mathrm{d} y \\
= & \int_{\partial \tilde{\phi}(\Omega)} \frac{\partial \Delta^{s} v_{1}}{\partial \nu} \frac{\partial \Delta^{s} v_{2}}{\partial \nu} \zeta \cdot \nu \mathrm{d} \sigma+\int_{\tilde{\phi}(\Omega)} \nabla \Delta^{s} v_{1} \nabla \Delta^{s} v_{2} \operatorname{div} \zeta \mathrm{d} y \\
& -\int_{\tilde{\phi}(\Omega)}\left(\Delta^{s+1} v_{1} \nabla \Delta^{s} v_{2}+\Delta^{s+1} v_{2} \nabla \Delta^{s} v_{1}\right) \cdot \zeta \mathrm{d} y
\end{aligned}
$$

By integrating by parts, changing variables in integrals and using formula (5.1), we obtain

$$
\begin{aligned}
\int_{\tilde{\phi}(\Omega)} \nabla \Delta^{s} & v_{i} \nabla\left(\left(\left.d\right|_{\phi=\tilde{\phi}} \Delta_{\phi}^{s} u_{j}[\psi]\right) \circ \tilde{\phi}^{(-1)}\right) \mathrm{d} y \\
= & -\left.\int_{\Omega} \Delta_{\tilde{\phi}}^{s+1} u_{i} d\right|_{\phi=\tilde{\phi}} \Delta_{\phi}^{s} u_{j}[\psi]|\operatorname{det} \nabla \tilde{\phi}| \mathrm{d} x \\
= & -\int_{\tilde{\phi}(\Omega)}\left(\Delta^{s+1} v_{i} \nabla \Delta^{s} v_{j}-\Delta^{2 s+1} v_{i} \nabla v_{j}\right) \cdot \zeta \mathrm{d} y
\end{aligned}
$$

for all $i, j \in\{1,2\}$. Finally, formula (5.6) easily follows by combining formulas (5.9)-(5.11) and by observing that $\frac{\partial \Delta^{s} v_{i}}{\partial \nu}=\frac{\partial^{2 s+1} v_{i}}{\partial \nu^{2 s+1}}$ on $\partial \tilde{\phi}(\Omega)$ since $v_{i} \in W_{0}^{2 s+1,2}(\tilde{\phi}(\Omega))$.

Acknowledgements. The authors are thankful to the referee for useful comments. The second author acknowledges financial support from the research project PRIN 2008 "Aspetti geometrici delle equazioni alle derivate parziali e questioni connesse". 


\section{REFERENCES}

[1] S. Agmon, Lectures on elliptic boundary value problems. Van Nostrand Mathematical Studies, No. 2 D. Van Nostrand Co., Inc. Princeton, N.J., Toronto London (1965).

[2] M.S. Ashbaugh and R.D. Benguria, On Rayleigh's conjecture for the clamped plate and its generalization to three dimensions. Duke Math. J. 78 (1995) 1-17.

[3] M.S. Ashbaugh and D. Bucur, On the isoperimetric inequality for the buckling of a clamped plate. Z. Angew. Math. Phys. 54 (2003) 756-770.

[4] D. Bucur and G. Buttazzo, Variational methods in some shape optimization problems. Appunti dei Corsi Tenuti da Docenti della Scuola, Notes of Courses Given by Teachers at the School. Scuola Normale Superiore, Pisa (2002).

[5] V. Burenkov and P.D. Lamberti, Sharp spectral stability estimates via the Lebesgue measure of domains for higher order elliptic operators. Rev. Mat. Comput. 25 (2012) 435-457.

[6] V. Burenkov and P.D. Lamberti, Spectral stability of higher order uniformly elliptic operators. Sobolev spaces in mathematics. II, in vol. 9 of Int. Math. Ser. (NY). Springer, New York (2009) 69-102.

[7] V. Burenkov, P.D. Lamberti and M. Lanza de Cristoforis, Spectral stability of nonnegative selfadjoint operators. Sovrem. Mat. Fundam. Napravl. 15 (2006) 76-111, in Russian. English transl. in J. Math. Sci. (NY) 149 (2008) 1417-1452.

[8] G. Buttazzo and G. Dal Maso, An existence result for a class of shape optimization problems. Arch. Rational Mech. Anal. 122 (1993) 183-195.

[9] R. Dalmasso, Un problème de symétrie pour une équation biharmonique. (French). A problem of symmetry for a biharmonic equation. In vol. 11 of Ann. Fac. Sci. Toulouse Math. (1990) 45-53.

[10] D. Daners, Domain perturbation for linear and semi-linear boundary value problems. Handbook of differential equations: stationary partial differential equations. Handb. Differ. Equ. VI. Elsevier/North-Holland, Amsterdam (2008) 1-81.

[11] F. Gazzola, H.-C. Grunau and G. Sweers, Polyharmonic boundary value problems. Positivity preserving and nonlinear higher order elliptic equations in bounded domains. Lect. Notes Math. Springer Verlag, Berlin (2010).

[12] P. Grinfeld, Hadamard's formula inside and out. J. Optim. Theory Appl. 146 (2010) 654-690.

[13] J.K. Hale, Eigenvalues and perturbed domains. Ten mathematical essays on approximation in analysis and topology. Elsevier B.V., Amsterdam (2005) 95-123.

[14] A. Henrot, Extremum problems for eigenvalues of elliptic operators. Frontiers in Mathematics. Birkhäuser Verlag, Basel (2006).

[15] D. Henry, Perturbation of the boundary in boundary-value problems of partial differential equations. With editorial assistance from Jack Hale and Antonio Luiz Pereira. In vol. 318 of London Math. Soc. Lect. Note Ser. Cambridge University Press, Cambridge (2005).

[16] D. Henry, Topics in Nonlinear Analysis, in vol. 192 of Trabalho de Mathemãtica. Universidade de Brasilia, Departamento de Matemãtica-IE (1982).

[17] B. Kawohl, Some nonconvex shape optimization problems. Optimal shape design (Tróia, 1998). In vol. 746 of Lect. Notes Math. Springer, Berlin (2000) 49-02.

[18] S. Kesavan, Symmetrization and applications. In vol. 3 of Ser. Anal. World Scientific Publishing Co. Pte. Ltd., Hackensack, NJ (2006).

[19] P.D. Lamberti and M. Lanza de Cristoforis, Critical points of the symmetric functions of the eigenvalues of the Laplace operator and overdetermined problems. J. Math. Soc. Japan 58 (2006) 231-245.

[20] P.D. Lamberti and M. Lanza de Cristoforis, A real analyticity result for symmetric functions of the eigenvalues of a domain dependent Dirichlet problem for the Laplace operator. J. Nonlinear Convex Anal. 5 (2004) 19-42.

[21] P.D. Lamberti and M. Lanza de Cristoforis, An analyticity result for the dependence of multiple eigenvalues and eigenspaces of the Laplace operator upon perturbation of the domain. Glasg. Math. J. 44 (2002) 29-43.

[22] E. Mohr, Über die Rayleighsche Vermutung: unter allen Platten von gegebener Fläche und konstanter Dichte und Elastizität hat die kreisförmige den tiefsten Grundton. Ann. Mat. Pura Appl. 104 (1975) 85-122.

[23] N.S. Nadirashvili, Rayleigh's conjecture on the principal frequency of the clamped plate. Arch. Rational Mech. Anal. 129 (1995) $1-10$.

[24] J.H. Ortega and E. Zuazua, Generic simplicity of the spectrum and stabilization for a plate equation. SIAM J. Control Optim. 39 (2001) 1585-1614.

[25] E. Oudet, Numerical minimization of eigenmodes of a membrane with respect to the domain. ESAIM: COCV 10 (2004) $315-330$.

[26] G. Pólya and G. Szegö, Isoperimetric Inequalities in Mathematical Physics. In vol. 27 of Ann. Math. Studies. Princeton University Press, Princeton, NJ (1951).

[27] G. Szegö, On membranes and plates. Proc. Nat. Acad. Sci. USA 36 (1950) 210-216.

[28] N.B. Willms, An isoperimetric inequality for the buckling of a clamped plate, Lecture at the Oberwolfach meeting on "Qualitiative properties of PDE" organized, edited by H. Berestycki, B. Kawohl and G. Talenti (1995).

[29] S.A. Wolf and J.B. Keller, Range of the first two eigenvalues of the Laplacian. In vol. 447 of Proc. Roy. Soc. London Ser. A (1994) 397-412. 